\title{
EFFECT OF A LOCALIZED MAGNETIC PERTURBATION \\ ON MAGNETIC ISLANDS IN A CYLINDRICAL PLASMA
}

\author{
Glenn Bateman \\ R. N. Morris \\ School of Nuclear Engineering \\ Georgia Institute of Technology \\ Atlanta, GA 30332
}

May 1980

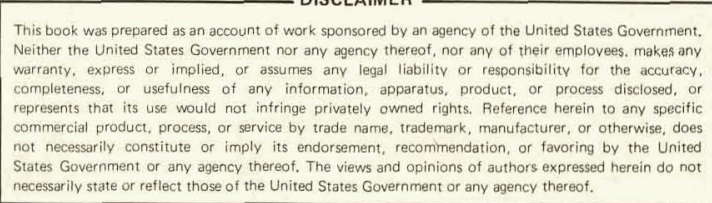

DISTRIBUTION OF THIS DOCUMENT IS UMLMMTED 


\section{DISCLAIMER}

This report was prepared as an account of work sponsored by an agency of the United States Government. Neither the United States Government nor any agency Thereof, nor any of their employees, makes any warranty, express or implied, or assumes any legal liability or responsibility for the accuracy, completeness, or usefulness of any information, apparatus, product, or process disclosed, or represents that its use would not infringe privately owned rights. Reference herein to any specific commercial product, process, or service by trade name, trademark, manufacturer, or otherwise does not necessarily constitute or imply its endorsement, recommendation, or favoring by the United States Government or any agency thereof. The views and opinions of authors expressed herein do not necessarily state or reflect those of the United States Government or any agency thereof. 


\section{DISCLAIMER}

Portions of this document may be illegible in electronic image products. Images are produced from the best available original document. 
NOTICE

This report was prepared as an account of work sponsored by an agency of the United States Government. Neither the United States Government nor any agency thereof, nor any of their employees, makes any warranty, express or implied, or assumes any legal liability or responsibility for the accuracy, completeness, or usefulness of any information, apparatus, product, or process disclosed, or represents that its use would not infringe privately owned rights. Reference herein to any specific commercial product, process, or service by trade name, trademark, manufacturer, or otherwise, does not necessarily constitute or imply its endorsement, recommendation, or favoring by the United States Government or any agency thereof. The views and opinions of authors expressed herein do not necessarily state or reflect those of the United States Government or any agency thereof. 


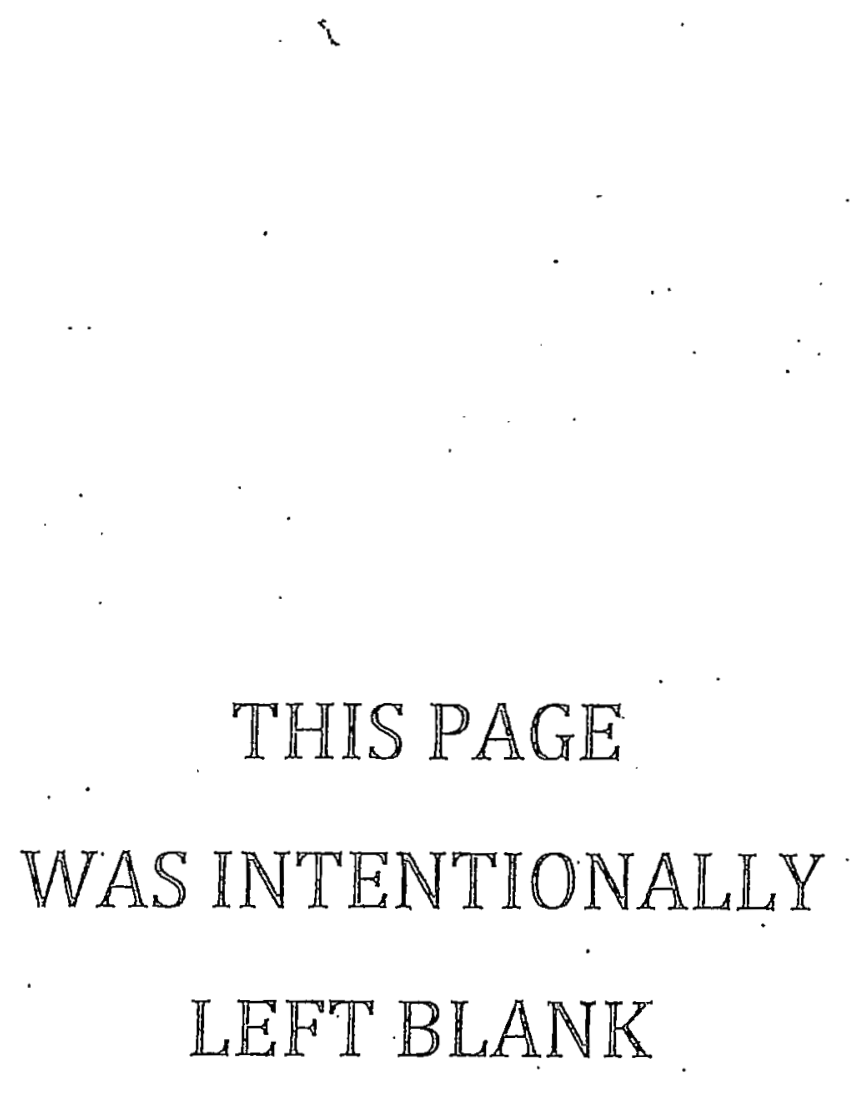




\begin{abstract}
A self-consistent plasma equilibrium model is developed to study the width of ergodic regions and magnetic islands in a periodic cylindrical plasma under the influence of a localized magnetic perturbation, such as that produced by a bundle divertor or ripple coil set. It is found that localized perturbations tend to produce poloidally symmetric annular ergodic regions and poloidally elongated magnetic islands rather than simple magnetic islands. Our plasma model takes into account the flattening of the current profile across each annular ergodic region and the concommitant steepening of the current profile between ergodic regions. Using current profiles inferred from experimental data, saturated tearing mode amplitudes are computed and found to agree with the experimentally observed Mirnov oscillation amplitudes. As the applied magnetic perturbation is turned on and increased, it is observed that the steepened current profile and resulting enhancement of tearing modes produces wider ergodic regions than would be expected from the vacuum magnetic perturbation alone.
\end{abstract}




\section{INTRODUCTION}

Non-axisymmetric magnetic perturbations in tokamaks are produced by many sources including bundle divertors [1-6], ripple coils [7], helical coils such as those used on PULSATOR [8-9], discrete toroidal field coils [10] as well as tearing modes [11-14] and other naturally occuring instabilities [15-16]. As a direct effect, these magnetic perturbations produce varying amounts of field strength ripple, magnetic islands, ergodic regions, and distortion of the plasma shape. These changes in the magnetic field, in turn, may enhance particle loss (particularly fast particles or trapped particles), may degrade energy confinement (particularly through electrons), and may alter the evolution of plasma instabilities (by changing the plasma profile and producing a disruptive instability, for example). It is particularly important to understand these effects when a deliberate attempt is made to impose a large non-axisymmetric perturbation on a tokamak plasma, as in the case of a bundle divertor, in order to optimize or minimize the effect of the perturbation.

This paper is concerned with the effect produced by the localized magnetic perturbation from a bundle divertor on ergodic regions and magnetic islands within the plasma. While previous studies $[1,4]$ have used a non-self-consistent model in which the vacuum field from the bundle divertor is added to the equilibrium plasma magnetic field, the present study develops a more self-consistent equilibrium model to accounl for the response of the plasma to the bundle divertor field. This model includes the effect of image currents within the plasma, the effect of naturally occurring magnetic islands due to tearing modes, and 
steepening of the current profile as the widths of the magnetic islands. or regions of ergodicity are increased by the bundle divertor perturbation. The basic equilibrium model is applied to a cylindrical plasma with periodic end conditions, perturbed by a simple bundle divertor, in the present study.

Some results from field line following using a vacuum magnetic perturbation are presented in section 1, before the self-consistent equilibrium model is developed in section 2, and applied in section 3 .

\section{OBSERVATIONS FROM A FIELD LINE FOLLOWING CODE}

Some observations based on results from a field line following computer code, similar to those reported in Refs. [17-19], will be reported here.

The field line following code integrates the equations

$$
\begin{aligned}
& \frac{d r(z)}{d z}=\frac{B_{r}(r, \theta, z)}{B_{z}(r, \theta, z)} \\
& \frac{d \theta(z)}{d z}=\frac{B_{\theta}(r, \theta, z)}{r B_{z}(r, \theta, z)}
\end{aligned}
$$

given the magnetic field components $\left(B_{r}, B_{\theta}, B_{z}\right)$ in a periodic cylinder. The location of a given field line $(x, \theta)$ in a cross-section of the plasma is plotted as a point after each successive integration along the periodicity length $2 \pi R$. Typically 500 points (corresponding to 500 transits) are plotted for a representative field line in order to exhibit magnetic islands or ergodic regions. After trying a number of differential equation solvers, it was found that the Bulirsch-Stoer 
extrapolation method used in the IMSL [20], subroutine DREBS was best for the high accuracy needed in this problem. In some cases, a relative error as small as $10^{-10}$ was needed for good convergence, particularly for very localized magnetic field perturbations. The machine epsilon (the smallest number that can be distinguished from unity) is about $3 \times 10^{-15}$ on our CYBER 70 computer.

Given a single helical perturbation with radial magnetic field

$$
B_{r}=B_{r 1}(r) \cos (m \theta-k \cdot z),
$$

where $k=n / R$, a simple analytic estimate for the width of a magnetic island is [14]

$$
W=4 r\left(\left|\frac{{ }_{r l}}{{ }^{B B}} \frac{q}{r q^{\prime}}\right|\right)^{1 / 2},
$$

where $q(r)=r B_{z} / R B_{\theta}$ and $r=r_{s}$ is the radius of the mode rational surface on which $q(r)=m / n$. In an effort to check the validity of Eq. (4), the field line following code was used with the vacuum magnetic ficld from eurrent flowing in a wire alung the axis of the cylinder (which yields a high shear magnetic field) and ${ }_{r l}$ proportional to $r$. It was found that the magnetic island width is a linear function of the square root of $B_{I l}$ which exceeds the estimate of Eq. (4) by only $2 \%$ when the width is two tenths of the minor radius and by only $4 \%$ when $\mathrm{W} / \mathrm{a} \approx$ -3. Hence Eq: (4) provides a remarkably good estimate of the magneticc island width even for large islands.

When two helical harmonics with the same mode rational surface are considered, as for example $\mathrm{m} / \mathrm{n}=2 / 1$ and $4 / 2$, we find that the 
resulting magnetic island width can be determined from Eq. (4) by adding together the $\mathrm{B}_{\mathrm{rl}} / \mathrm{m}$ for each individual harmonic. In other words, the squares of the island widths are additive. The structure of the islands make a continuous transition from $m=2$ to $m=4$.

When two incommensurate harmonics with overlapping magnetic islands are considered, as for example $\mathrm{m} / \mathrm{n}=2 / 1$ with width $\mathrm{W}=.14$ and $\mathrm{m} / \mathrm{n}=$ $7 / 4$ with $W=.092$, we also find that the squares of the individual widths are additive, so that $\mathrm{Eq} \cdot$ (4) can be used if $\mathrm{B}_{\mathrm{rl}} / \mathrm{m}$ are added together from each harmonic. However, the magnetic islands do not deform continuously as in the last case. With even small amounts (10\%) of the $7 / 4$ helical perturbation, the $x$-points of the $2 / 1$ magnetic islands become fuzzy with what appears to be ergodic behavior [21]. When the amplitudes of the perturbations become comparable, the distinct islands give way to a lumpy annular region of apparantly ergodic magnetic field. This persists until $\mathrm{B}_{\mathrm{r} 1} / \mathrm{m}$ for the $2 / 1$ perturbation is substantially smaller (much less than $10 \%$ ) of $\mathrm{B}_{\mathrm{rl}} / \mathrm{m}$ for the $7 / 4$ perturbation. The presence of many incommensurate helical magnetic field perturbations simultaneously can be expected to make the annular ergodic regions more cylindrically symmetric -- less bumpy -- which can be used to simplify our mudel for the transport-induced current profile, as described in section 2.

The perturbations just considered are sums of helical perturbations, each with $\cos (m \theta-k z)$ dependence. Now consider a perturbation which is localized within a sector $-\theta_{\mathrm{m}}<\theta<\theta_{\mathrm{m}},-z_{\mathrm{m}}<z<z_{\mathrm{m}}$, 


$$
\begin{aligned}
& B_{r}^{1}=B_{r 1} r \theta\left(\theta_{m}^{2}-\theta^{2}\right)\left(z_{m}^{2}-z^{2}\right)^{2} \\
& B_{\theta}^{1}=\frac{1}{2} B_{r l} r\left(\theta_{m}^{2}-\theta^{2}\right)^{2}\left(z_{m}^{2}-z^{2}\right)^{2} . \\
& B_{z}^{1}=0,
\end{aligned}
$$

and identically zero everywhere else, together with a plasma equilibrium field consistent with the current profile

$$
J_{z}(r)=J_{z 0}\left(1-r^{2}\right)^{2} \text { for } 0<r<1 \text {, }
$$

adjusted so that $1<q(r)<3$ within the plasma given $R=3$. This perturbation has smooth first derivatives and satisfies $\nabla \cdot \nabla^{1}=0$. The radial component reverses sign at $\theta=0$ as illustrated in Fig. 2 . The effect of this perturbation on magnetic islands depends on the sign of the radial perturbation relative to the helicity of the equilibrium. field, as illustrated for an $\mathrm{m} / \mathrm{n}=2 / 1$ island in Figs. $2 \mathrm{a}$ and $2 \mathrm{~b}$. For the orientation shown in Fig. $2 a$, field lines just off the $q=2$ mode rational surface that drift into the perturbation sector are pushed through the rational surface and drift back out in the opposite direction. to form a poloidally elongated magnetic island with $\mathrm{x}$-points at $\theta=0^{\circ}$ and $\theta=180^{\circ}$ (the nature of the mapping at the $q=2$ surface forces the top to bottom reflection symmetry even though the perturbation is localized at only the top in Fig. 2.). For the orientation shown in Fig. $2 b$, field lines in the neighborhood of $\theta=0$ (and $\theta=180^{\circ}$ by reflection) near the $q=2$ surface form small magnetic islands with 0 -points at $\theta=0^{\circ}$ and $180^{\circ}$. Field lines exactly on the $q=2$ surface outside of the perturbation sector are left completely unperturbed (e.g. the isolated dots in Fig. $2 \mathrm{~b}$ ). Hence, a localized magnetic 
perturbation, similar to that produced by the horizontal legs of a bundle divertor or ripple coil, will produce poloidally elongated magnetic islands as well as smaller structures.

Figs. $2 a$ and $2 b$ were produced by following a selection of field lines which were inside magnetic islands. Field lines outside magnetic islands are characterized by a considerable amount of ergodicity, particularly as the localized perturbation amplitude is increased, as shown in Fig. 3. The annular ergodic regions become smoother -- more cylindrically symmetric -- as the perturbation is made more localized. This is consistent with the fact that more localized perturbations contain a broader spectrum of helical harmonics which, in turn, produce more overlap among magnetic islands. Note that all the radial drift of the field lines shown in Fig. 3 occurs within the localized sector $|\theta|<0.25$ radians and $|z|<1$. It was found necessary to integrate with a relative error as low as $10^{-10}$ for these computations.

\section{PERTURBED EQUILIBRIUM}

Computational studies of the ergodic regions and magnetic islands resulting from bundle divertors or similar localized magnetic perturbations have generally used a non-self-consistent model in which the vacuum magnetic perturbation from the bundle divertor coil is added to the unperturbed plasma equilibrium magnetic field, as illustrated in the previous section of this paper. It is the purpose of this section to develop elements of a self-consistent model in which image currents and deformation of the current channel within the plasma may have an effect on the width of those ergodic regions and magnetic islands that are driven by the externally applied perturbation, as well as those which occur naturally due to tearing modes. This work is an extension of some 
methods used recently by Carreras et al. [13] to predict saturated magnetic island widths (and Mirnov oscillation amplitudes) due to tearing modes and externally applied helical drivers.

It is useful to split off and consider separately the most prominant of those helical harmonies of the magnetic perturbation

$$
\operatorname{Re}\left[B_{1}(x) \exp i(m \theta-k z)\right]
$$

with resonances within the plasma, $n q(r)-m=0$, where $k=n / R, m$ and $\mathrm{n}$ are the poloidal and longitudinal mode numbers, $\mathrm{q}=\mathrm{rB} / \mathrm{RB}$, and $2 \pi \mathrm{R}$ is the periodicity length of the cylindrical plasma being considered. The rest of the magnetic perturbation can be treated as a vacuum magnetic field, relatively unaffected by image currents within the plasma or other self-consistent effects. For example, perturbed plasma currents are not expected to significantly alter the magnetic field ripple or that part of the magnetic field in the bundle divertor chamber outside the main body of the plasma.

In steady state, Maxwell's equations $\nabla \cdot \vec{B}=0$ and $\mu_{0} J=\nabla \times \vec{B}$ imply, for each helical harmonic,

$$
\begin{aligned}
& { }_{i B}=\left[-m\left(r B_{r 1}\right)^{\prime}+k r^{2} \mu_{0} J_{r l}\right]^{/\left(m^{2}+k^{2} r^{2}\right)} \\
& i_{z 1}=\left[k r\left(r B_{r 1}\right)^{\prime}+m r \mu_{0} J_{r l}\right] /\left(m^{2}+k^{2} r^{2}\right) \\
& {\left[r\left(r B_{r 1}\right)^{-} /\left(m^{2}+k^{2} r^{2}\right)\right]^{\prime}-B_{r 1}} \\
& =r\left[\frac{2 m k r \mu_{0} J_{r l}}{m^{2}+k^{2} r^{2}}-k r i_{\mu_{0}} J_{\theta 1}-m i \mu_{0} J_{z l}\right] /\left(m^{2}+k^{2} r^{2}\right)
\end{aligned}
$$


Eq. (7) is used to determine the contribution from any given configuration of coil currents to the particular solution of a helical harmonic radial magnetic field. For example, from a simple pair of bundle divertor coils curved along an arc of constant radius, ${ }{ }_{c}$, the jump in the radial derivative of $B_{r}$ from $r_{c}-\varepsilon$ to $r_{c}+\varepsilon$, in the large aspect ratio limit $(\mathrm{kr} \ll \mathrm{m})$, is

$$
\left[\frac{r\left(r B_{r l}\right)^{\prime}}{m^{2}+k^{2} x^{2}}\right]=\frac{2 i \mu_{o} I}{\pi^{2} m n} \sin \left(m \theta_{\max }\right) \sin ^{2}\left(k z_{\max }\right)
$$

where $I$ is the current in each coil leg and the coils subtend the arc $-\theta_{\max }<\theta<\theta_{\max }$ and length $-z_{\max }<z<z_{\max }$, as illustrated in Fig. 4 .

Now consider a force-free perturbed equilibrium

$$
J \times \vec{B}=0
$$

Most of the current in a tokamak is force-free $(\vec{J}$ is mostly parallel to B) even when $B\left(\equiv 2 \mu_{0} p / B^{2}\right)$ is several percent. The parallel component of the plasma current contributes most to shear and magnetic structure, particularly near mode rational surfaces. Ergodic regions cannot support a steady state'pressure gradient in any event. This force-free equilibrium assumption can be relaxed in future work if need be: Note that the force-free equilibrium Eq. (9) together with $\nabla \cdot \vec{B}=0$ implies that $J / B$ is uniform along field lines.

If Eq. (y) is linearized for a helical harmonic perturbation about a cylindrically symmetric equilibrium $\left(B_{\theta 0}(r), B_{z O}(r)\right)$ and $E_{4} \cdot(5)$ and (6) are used to eliminate $B_{\theta 1}$ and $B_{z 1}$, the equation for the radial magnetic perturbation is 


$$
\begin{aligned}
& \left(k r B_{z 0}-m B_{\theta 0}\right)\left\{\left[r\left(r B_{r 1}\right)^{-} /\left(m^{2}+k^{2} r^{2}\right)\right]^{-}-B_{r 1}\right\} \\
& +\left\{r^{2}\left[m\left(r B_{\theta 0}\right)^{\prime} / r\left(m^{2}+k^{2} r^{2}\right)\right]^{\prime}+\left[k r^{2} B_{\theta 0}\left(r B_{\theta 0}\right)^{-} / B_{z 0}\left(m^{2}+k^{2} r^{2}\right)\right]-B_{r l}=0 .\right. \\
& \text { and } \\
& \mathrm{J}_{\mathrm{r} 1}=\mathrm{J}_{\mathrm{z} 0} \mathrm{~B}_{\mathrm{r} 1} / \mathrm{B}_{\mathrm{z} 0}
\end{aligned}
$$

In the large aspect ratio limit $\mathrm{kr} \ll \mathrm{m}$ and $\mathrm{B}_{\theta 0} \ll \mathrm{B}_{20}, \mathrm{Eq} \cdot(10)$ becomes

$$
\left[r\left(r B_{r l}\right)\right]^{\circ}-m^{2} B_{r l}+m r^{2}\left[\left(r B_{\theta 0}\right)^{\prime} / r\right] B_{r l} /\left(k r B_{z 0}-m B_{\theta 0}\right)=0
$$

Eq. (10) or (12) is to be solved with boundary conditions $B_{r 1} \propto r^{m}-1$ at $\mathrm{r}=0$ and $\mathrm{B}_{\mathrm{rl}}=0$ at a perfectly conducting wall or at infinity, together with the contributions from applied coil currents (Eq. 7).

\section{Profile with Flat Spots}

It is evident that Eqs. (10) and (12) are singular at the radius of the mode rational surface $r_{s}$ where

$$
k_{s} B_{z 0}\left(r_{s}\right)-m B_{\theta 0}\left(r_{s}\right)=B_{\theta 0}\left(r_{s}\right)\left(n q\left(r_{s}\right)-m\right)=0
$$

However, this singularity is removed if the current profile is flattered in the neighborhood of the mode rational surface -- i.e.,

$$
\mu_{0} J_{z 0}=\left[\left(r B_{\theta 0}\right)^{\prime} / r\right]^{-}=0 \text { at } r=r_{s}
$$

in the large aspect ratio limit.

The flattening of the current profile is consistent with the parallel component of Ohm's law

$$
\eta \vec{J} \cdot \vec{B}=\vec{E} \cdot \vec{B}=E_{7} B_{7},
$$


with uniform $\mathrm{E}_{z}$ (in steady state) when there is a poloidally symmetric ergodic region (producing uniform electron temperature and, therefore, uniform parallel resistivity $n$ ) in the neighborhood of a mode rational surface. The study of poloidally symmetric ergodic regions is particularly simple since the current profile within each ergodic region is flat (the last term in Eq. (12) is zero) while the current profile between the ergodic regions is determined by the temperature (resistivity) profile together with Eq. (15). With flat spots in $\mathrm{J}_{z 0}(r)$, Eq. (12) is no longer singular and the linearization of Eq. (9) could be expected to be reasonable. The study of nonlinear effects, other than the flattening of the poloidally symmetric current profile, has led to no further qualitative changes in this model.

For arbitrary ergodic region width around a given mode rational surface, Eq. (12) generally does not have a solution satisfying the boundary conditions. However, a solution can be found by varying the width (and the resulting current profile) until the proper value is found. Effectively the width plays the role of an eigenvalue in Eq-(12). The width of the ergodic region, in turn, is related to the amplitude of the radial magnetic perturbation, by Eq. (4) as described in the last section, which is varied by varying the amplitude of the homogeneous solution of Eq. (12). This is the algorithm we use in this paper to determine the self-consistent solution for a selection of helical harmonics driven by a localized perturbation.

Effectively, this algorithm is an extension of the one used by Carreras et al. [13] to match the experimentally observed Mirnov oscillation amplitude given a model for the experimentally inferred current profiles in ORMAK and $T-4$. Our algorithm is more flexible in that it 
can handle more than one harmonic with the same mode rational surface (e.g. $m / n=2 / 1,4 / 2,6 / 3, \ldots)$ and we can study the simultaneous effect of several ergodic regions on the overall current profile. (Other things remaining equal, the current gradients must become steeper if the flat regions become wider.) Our algorithm is more difficult to implement because the current profile is affected by the widths of the ergodic regions, which are determined iteratively.

\section{Linear Ramp Model}

We have implemented the algorithm just described by approximating the current profile by a piecewise linear function of the plasma radius, called a linear ramp model, as illustrated in Fig. 5. Using this approximation, the poloidal magnetic field and longitudinal current density have the form

$$
\begin{aligned}
& B_{\theta}(r)=B 2(N) r^{2}+B 1(N) r+B M 1(N) / r \\
& \mu_{0} J_{z}(r) \equiv \frac{1}{r}\left(r B_{\theta}\right)^{-}=3 \cdot B 2(N) \cdot r+2 \cdot B 1(N)
\end{aligned}
$$

where $B 2(N), B 1(N)$ and $B M 1(N)$ are coefficients to be determined in each region $N=1,2, \ldots$ from the center of the plasma to the wall. The current gradient, $\mu_{0} J_{z}^{\prime}(r)=3 B 2(N)$, is zero in each flat region ( $N$ odd) and $B 2(N)$ may be prescribed with a different value in each sloped region ( $N$ even) or it may be computed, with each iteration of the island widths, by an algorithm designed to hold the edge of the currentcarrying region REDGE fixed. In the first region we have $B M 1(1)=0$ (to avoid singular $B_{\theta}$ at $r=0$ ). $B I(1)$ is prescribed or related to the q-value on axis 


$$
\mathrm{Bl}(1)=\mathrm{B}_{\mathrm{z}} / \mathrm{Rq}(\mathrm{r}=0)
$$

and RQ1 is prescribed. The rest of the coefficients are determined by requiring continuity for $B_{\theta}(r)$ and $\mu_{0} J_{z}(r)$ between each region and by computing the position of each mode rational surface

$$
q(r)=r B_{z} / R B_{\theta}(r)=m / n \text { for } r=R S L(N)
$$

self-consistently given the half-width HWL(N) of each flat spot (corresponding to a magnetic island or ergodic region), working from the center to the edge. Hence, the radii of the break points $R B(N)$ which bound the linear segments are determined self-consistently along with the radii of the mode rational surfaces.

Once the equilibrium coefficients and breakpoints are computed for any given set of island widths, the radial magnetic field perturbation equation (Eq. 12) for each mode is integrated, out from the center and in from the coils to the mode rational surface; where the amplitude of $B_{r 1}$ is. made consistent with Eq. (4). The island widths are iterated until solutions with continuous radial derivative are found for all the modes $B_{r l}(N)$ being considered. It was found that the Runge-Kutta-Verner differential equation solver (subroutine DVERK in IMSL [20]) did not converge well when solving Eq. (12) for small island widths. The Adams method in subroutine DGEAR [20], however, works quite well (we normally use a tolerance of $10^{-5}$ ). Newton's method [22] is used together with a set of constraints to force the discontinuity in the radial derivative of each mode $\left(\Delta^{2}[11-14]\right)$ to zero by iterating on the island widths. 
Simple Magnetic Islands

When simple magnetic islands are present, rather than poloidally symmetric ergodic regions, the poloidally averaged current profile looks more like a point of inflection at the mode rational surface [12] rather than a completely flat spot. The gradient of the poloidally averaged current near the mode rational surface must be calculated carefully, since the singularity in Eq. (8) enhances its effect. The corrections needed to study simple magnetic islands as well as the corrections due to the in-out asymmetry of a magnetic island in cylindrical geometry will be calculated in this section.

The helical stream function $\psi_{*}[14], \nabla \psi_{*} \times \hat{z}=\vec{B}_{*}$, is derived by subtracting a zero shear magnetic field for the mode rational surface being considered from the true magnetic field

$$
B_{* \theta}=B_{\theta}(r)-B_{\theta}\left(r_{s}\right) r / r_{s}
$$

Using $B_{\theta}=B l \cdot r+B M l / r$ and the observed radial magnetic field

$$
B_{r}(r) \simeq B_{r 1}\left(r / r_{s}\right)^{-m-1} \sin (m \theta-k z)
$$

the helical stream function becomes

$$
\begin{aligned}
& \Psi_{*}=B M 1 \cdot\left[-\ln \left(r / r_{s}\right)+\frac{1}{2} r^{2} / r_{s}^{2}\right]-B_{r l}\left(r^{-m} / m r_{s}^{-m}-1\right) \cos (m \theta-k z) \\
& =B M 1 \cdot\left[\frac{1}{2}+x^{2}-\frac{1}{3} x^{3}+\ldots\right]-\frac{r_{s}{ }^{B} 1}{m}(1+x)^{-m}\left[1-2 \sin ^{2} \frac{1}{2}(m \theta-k z)\right]
\end{aligned}
$$

where $r \equiv r_{0}(1+x)$. The separatrix is given by

$$
\psi_{*}(x, \theta)=\psi_{s} \equiv \frac{1}{2} B M 1-r_{s}{ }_{r 1} / m
$$

and the half-width by 


$$
H W=H_{0} \sqrt{\frac{(1+x)^{-m}}{\frac{1}{2}-[\ln (1+x)-x] / x^{2}}} .
$$

where $\mathrm{HW}_{\mathrm{O}}=\mathrm{W} / 2$ is given by Eq. (4), and Eq - (24) should be iterated using $\mathrm{x}= \pm \mathrm{HW} / \mathrm{r}_{\mathrm{s}}$ for the outer $(+)$ or inner $(-)$ edge of the island.

Now consider the current profile. Assuming a source of heat near the center of the plasma and neglecting Ohmic heating, the temperature profile and therefore the current profile will be uniform within each magnetic island. The current density will be a function of $\psi_{\star}$, $\mathrm{J}_{z}=\mathrm{J}_{z}\left(\Psi_{*}\right)$, elsewhere. Assuming the current density is a linear function of the radius along a ray passing through the widest part of the island

$$
\left.\mathrm{J}_{z}(\mathrm{r})=\mathrm{J}_{\mathrm{z}}\left(\mathrm{r}_{\mathrm{s}}\right)-\left|\mathrm{J}_{\mathrm{zo}}\right|^{\circ} \mathrm{x}-\mathrm{HW}\right) \text { along } \mathrm{m} \theta-\mathrm{kz}=\pi,
$$

then the current density outside of the island is

$$
\mathrm{J}_{z}(\psi)=\mathrm{J}_{z}\left(\mathrm{r}_{\mathrm{s}}\right)-\left|\mathrm{J}_{\mathrm{zO}}^{-}\right|\left[\sqrt{\left(\psi-\psi_{s}\right) / \mathrm{BM} 1+\mathrm{HW}^{2}}-\mathrm{HW}\right]
$$

Now determine the poloidally averaged current density

$$
\bar{J}_{z} \equiv \frac{1}{2 \pi} \int_{-\pi}^{+\pi} d(m \theta) J_{z}(x, \theta)
$$

and the coefficient needed for the last term in Eq. (12)

$$
\frac{\mathrm{mr}^{2} \mu_{0} \bar{J}_{z}^{-}}{\mathrm{krB}_{z O}-\mathrm{mB}_{\theta O}}=\frac{r_{s}^{4}}{2 \cdot \mathrm{BMI}} \frac{\mu_{0} \bar{J}_{z}^{-}(r)}{\left(r-r_{s}\right)}
$$

After some algebra we find

$$
\frac{\mu_{0} \bar{J}^{-}(r)}{r-r}=\frac{\mu_{0} J^{\prime}}{H W} Z(u)
$$

where $\quad Z(u) \equiv \frac{2}{\pi} \int_{0}^{\left(\sin ^{-1} u, \pi / 2\right)} \frac{\mathrm{d} \theta}{\sqrt{u^{2}+\cos ^{2} \theta}}$ 
and $u \equiv\left(x-r_{s}\right) / H W$. The function $Z(u)$, shown in Fig. 6, may be reasonably approximated by

$$
z(u) \simeq\left\{\begin{array}{ll}
0.63 u & \text { for } u<1 \\
\frac{1}{2}\left(1-\frac{0.17}{u}\right) & \text { for } u>1
\end{array} .\right.
$$

We have implemented the corrections needed to consider a simple magnetic island [Eqs. (28) - (31)] and radial asymmetry [Eq. (24)] as an option in the linear ramp model. We find that these corrections increase the predicted island width by 30 to $60 \%$ and the predicted Mirnov oscillation amplitude by 50 to $80 \%$ when only the $\mathrm{m} / \mathrm{n}=2 / 1$ helical harmonic is considered alone and the profile is adjusted to hold RQI, $r_{s}$, and $q_{\text {edge }}$ fixed. The correction for radial asymmetry, taken alone, generally has a smaller effect. If Ohmic heating within the islands were included, the island widths would probably be larger still.

\section{RESULTS}

Comparison with Experimentally Observed Mirnov Oscillations

In order to check the validity of our model and computer code, and to establish realistic base cases for the subsequent study of the effect of a bundle divertor or similar localized perturbation, an attempt was made to match experimental data provided by M. Murakami and J. L. Dunlap from a series of particularly well documented discharges in ORMAK at ORNL during 1975 [23]. This data consists of Mirnov oscillation amplitudes $\left(\tilde{B}_{\theta} / B_{\theta}\right.$ at the wall) together with toroidal current density profiles inferred from measurements of electron temperature profile and total current. This current profile represents a time average over fluctuations caused by sawtooth oscillations and the 
rotating magnetic islands. It was not possible to make direct measurements of current profile or magnetic island widths within the plasma. The same data was used by Carreras et al. [13] as part of their comparison with experiment.

It was observed that the computed Mirnov oscillation amplitudes were quite sensitive to the position of the mode rational surface and to details of the current profile, particularly inside the radius of the mode rational surface. It was not possible to match the experimentally inferred current profiles point by point with our relatively crude linear ramp model. Even after matching the position of the mode rational surface and matching the q-value at the limiter and at the axis, there is still the freedom to choose RQ1, the radius of the first flat spot. The best results were obtained when RQI was chosen as large as possible consistent with the experimental data. This choice maximizes the current gradient within the $q=2$ surface. In particular, when sawtooth oscillations are present, it was best to choose RQl to be the maximum extent of the $q=1$ region just after the internal disruption. This is consistent with the observation in PULSATOR [24] that the amplitude of Mirnov oscillations and the probability of major disruption are maximum just after the minor disruption part of the sawtooth oscillation.

The results of this comparison are shown in Fig. 7, where the normalized Mirnov oscillation amplitude, $\widetilde{B}_{\theta} / B_{\theta}$ for $m / n=2 / 1$ is plotted as a function of the q-value at the limiter. Note, the computed poloidal field perturbation, $\tilde{B}_{\theta}=i B_{\theta l}$, is related to the radial field perturbation $\mathrm{B}_{\mathrm{rl}}$ by Eq. (5), where $\mathrm{J}_{\mathrm{rl}}=0$ from the edge of the plasma out. Here, it is assumed here that the wall coincides with the edge of 
the plasma. The computed results follow the same trend as the experimental data, but are too low, especially for large q-values. Computed values using the simple magnetic island model come closer to the experimental data than the poloidally symmetric ergodic annulus model. Possibly the inclusion of Ohmic heating within the magnetic islands would bring the results closer.

In a more recent implementation of the linear ramp model, the radius and $q$-value of the limiter and $q(r=0)$ are held fixed while the radius of the central uniform-current region is computed. Provision is made for islands to overlap and to eat into the edge of the current profile if necessary. The current profile is computed working from the edge to the center of the plasma with options for either fixed mode rational surfaces or fixed relative current slopes $(B 2(2 n))$ which must be adjusted by a computed scale factor to make $\mathrm{B}_{\theta}(\mathrm{r} \longrightarrow 0) \longrightarrow 0$. The new algorithm was found to be particularly useful for studying bundle divertors, where the magnetic structure determines the edge of the current profile. The + marks in Fig. 7 show the Mirnov oscillation amplitudes computed using the new alorithm with only the $\mathrm{m} / \mathrm{n}=2 / 1$ helical harmonic with fixed mode rational surface matching the experimentally inferred radius of the $y=2$ surface. In most cases, this procedure gave excellent agreement with experimental observations.

\section{Effect of a Bundle Divertor}

An example of the effect of a simple bundle divertor on the width of poloidally symmetric ergodic regions in a cylindrical plasma is described in this section. The bundle divertor being considered here consists of a pair of square-shaped coils curved along an arc of conetant radius, illustrated in Fig. 4, whose concribution to the helical 
harmonic field is described by Eq. (8). For this particular study, $z_{\max }=.8, r_{\text {COIL }}=1.2, r_{\text {WALL }}=3 ., r_{\text {COIL }} \cdot \theta_{\max }=.4$, the plasma radius and longitudinal field are normalized to unity while the aspect ratio was taken to be $R=3.57$ (to be consistent with ORMAK data). Significant divertor action is achieved with $\mu_{0} I / a B_{z}=.3$. Only the lowest order harmonics with resonances within the plasma will be considered here. The transition from a simple magnetic island produced by a saturated tearing mode (generally $\mathrm{m} / \mathrm{n}=2 / 1$ ) to a poloidally symmetric ergodic region was not studied. From the observations made at the end of section 2, it can be inferred that the contribution from the tearing mode will be reduced by 30 to $60 \%$ during this transition. Here, only poloidally symmetric ergodic regions will be considered.

As the current in the bundle divertor is increased, many changes happen simultaneously within the plasma as the ergodic regions grow wider and the current profile changes. Figure 8 shows the effect of the simple bundle divertor on the $\mathrm{m} / \mathrm{n}=2 / 1$ and $3 / 1$ helical harmonics, holding the positions of the mode rational surfaces fixed corresponding to the experimentally inferred profile with $q-1$ imiter $=4.2$ and $q(0)=1.0$ (second data point from left in Fig. 7). Figure 9 shows the same case with the more realistic assumption of fixed relative current slopes adjusted by a scale factor. The new algorithm, working from the edge to the center was used in both cases, with q-limiter (total plasma current) and $q(0)$ held fixed as the divertor is turned on. The computed half-widths of the ergodic regions are plotted as a function of bundle divertor current in the upper portions of Figs. 8 and 9. For comparison, the dashed curves indicate the half-widths produced by the vacuum magnetic perturbation for the $3 / 1$ and $2 / 1$ modes (they happen to be nearly identical in Fig. 8) using the same poloidal field $\left(B_{\theta}(r), q(r), q^{\prime}(r)\right.$ 
in Eq. (4)) as in the self-consistent calculation. The lower portion of Figs. 8 and 9 show the linear ramp current profiles with the bundle divertor off (solid line) and on (dashed line), with the experimentally inferred current profile (smooth curve, no divertor) shown for comparison.

In Fig. 8, with fixed mode rational surfaces, the self-consistently computed $3 / 1$ half-width is consistently wider than the vacuum field prediction, while the $2 / 1$ half-width drops slightly below the unperturbed value until it becomes comparable with the vacuum half-width, as the bundle divertior current is increased. This behavior can be understood by noting that the current profile between the $2 / 1$ and $3 / 1$ islands becomes steeper as the divertor is turned on, while the profile becomes less steep between the $2 / 1$ island and the center of the plasma. In general, the saturated tearing mode amplitude increases as the current profile becomes steeper on the inner edge or shallower on the outer edge of the magnetic island, and vice versa. Hence the changing current profile has enhanced the $3 / 1$ saturated tearing mode at the expense of the $2 / 1$ mode.

In the more realistic case where the relative slopes of the current profile are fixed, shown in Fig. 9, the $3 / 1$ island width remains nearly the same as that predicted by the vacuum perturbation while the $2 / 1$ width is approximately the sum of the vacuum width and the unperturbed saturated tearing mode width. The islands significantly erode the edge of the current profile and force the central $q=1$ region (sawtooth oscillation region) to expand in order to accommodate the same total plasma current. It was not possible to obtain convergence as the bundle 
divertor current was increased further, since the broader islands and correspondingly steeper current profile greatly enhanced the saturated tearing modes, which made the islands still broader and profile steeper, in a process analogous to the discuptive instability.

\section{CONCLUSIONS}

Several effects happen simultaneously when a localized magnetic perturbation is applied to a current-carrying plasma. A very localized perturbation tends to produce poloidally symmetric annular ergodic regions and poloidally elongated magnetic islands rather than simple magnetic islands. The poloidally averaged current profile is more completely flattened in each of these annular ergodic regions than it would be with simple magnetic islands. The saturated tearing mode amplitude is smaller with ergodic regions than with simple magnetic islands. The amplitude of saturated tearing modes depends sensitively on details of the current profile, increasing as the current gradient just inside the mode rational surface is increased or as the current gradient just outside the mode rational surface is decreased. As the applied perturbation is increased, the width of the ergodic regions and the current gradient between them increases, which enchances the saturated tearing mode amplitude for at least some of the modes. These changes in current profile and their effect on tearing modes appear to be more important than the direct effect of helical image currents in the plasma.

The most important change being planned in the algorithm is to replace Eq. (4) with a more realistic estimate of the ergodic region width when there are many harmonics with the same mode rational surface. 
Equation (4), when used with only the lowest order resonant harmonic, as done in the present study, probably overestimates the ergodic region width since the harmonics of a localized perturbation cancel almost everywhere. Studies done to date with up to 8 helical harmonics with different mode rational surfaces have indicated that higher harmonics (m $\geq 4$ ) tend to be well approximated by the vacuum perturbation. This should make it easier to determine the true widtins of ergodic regions by using a field line following code as in section 1.

Another improvement being considered is to use a transport code to determine the electron temperature profile and therefore obtain a better approximation to the current profile between ergodic regions, rather than relying on the ad hoc procedures used in the present study. Unfortunately, the electron heat conductivity and radiation loss mechanisms have so far been only crudely approximated by empirical models. Given the demonstrated sensitivity to current profile, it is not clear if there would be any advantage to studying these modes in toroidal geometry at this stage.

\section{Acknowledgments}

We would like to thank Drs. Masanori Murakami and Julian Dunlap for generously providing detailed experimental data taken on the ORMAK tokamak at Oak Ridge National Laboratory. We would like to dedicate this work to the memory of Dr. B. V. Waddell and to acknowledge the encouragement and suggestions from Drs. B. Carreras, H. R. Hicks, J. A. Kome, J. N. Davidson and W. M. Stacey. This research was supported by the U.S. Department of Energy under contract no. DE-AS05-78ET52025. 


\section{Figure Captions}

Fig. 1 Magnetic structure near the $q=2$ surface produced by two helical perturbations $\mathrm{m} / \mathrm{n}=2 / 1$ and $7 / 4$ in the cylindrical field. of a current filament at $r=0$ with aspect ratio 3 and $B_{z}=1$. Radial field perturbation amplitudes are. noted at the $q=2$ surface.

Fig. 2 Cross section of the $\mathrm{m} / \mathrm{n}=2 / 1$ magnetic island produced by a localized magnetic perturbation confined to the sector $|\theta|<1$ radian, $|z|<1$ with amplitude $\mathrm{B}_{\mathrm{rl}} / \mathrm{B}_{\mathrm{z} 0}=.04$ in (a) and $|\theta|<.5,|z|<1, \mathrm{~B}_{\mathrm{rl}} / \mathrm{B}_{\mathrm{z} 0}=-0.32$ in (b).

Fig. 3 Sample of the magnetic structure produced by a localized magnetic perturbation confined to the sector $|\theta|<0.25$ radians, $|z|<1$, with amplitude $\mathrm{B}_{\mathrm{rl}} / \mathrm{B}_{\mathrm{z} 0}=2.56$.

Fig. 4 Schematic of the simple bundle divertor coil set, used in this paper [Eq. 8], in the cylindrical surface $\mathbf{r}=$ constant.

Fig. 5 Schematic of the linear ramp current profile as a function of radius in a cylindrical plasma.

Fig. 6 Function $Z(u)$ defined by Eq. (30). 
Figure Captions

Page 2

Fig. 7 Computed $m / n=2 / 1$ Mirnov oscillation amplitudes at the limiter ( = wall radius here) compared with experimental data from ORMAK [23]. The $x$ and o points are computed using the best match to the experimentally inferred current profile while the + points are computed just matching the inferred radius of the $q=2$ surface.

Fig. 8 Effect of a simple bundle divertor (Fig. 4) on ergodic region half-widths (top) and current profile (bottom) holding the radii of the $q=2$ and $q=3$ surfaces and $q_{1 \text { imiter }}=4.2$ fixed. The dashed line at top are the nearly coincident halfwidths computed from the vacuum field perturbation. The smooth curve at bottom is the experimentally inferred current profile.

Fig. 9 Effect of a simple bundle divertor on ergodic region half-widths (top) and current profile (bottom) holding the relative slopes of the current profile and $q_{1 \text { imiter }}=4.2$ fixed. 


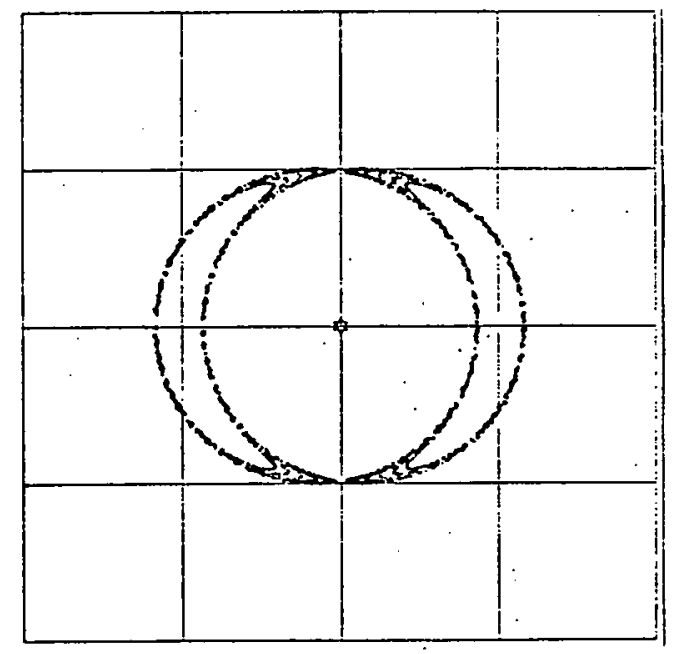

$$
\begin{aligned}
& \mathrm{B}_{\mathrm{r} 21}=.002 \\
& \mathrm{~B}_{\mathrm{r} 74}=.0
\end{aligned}
$$

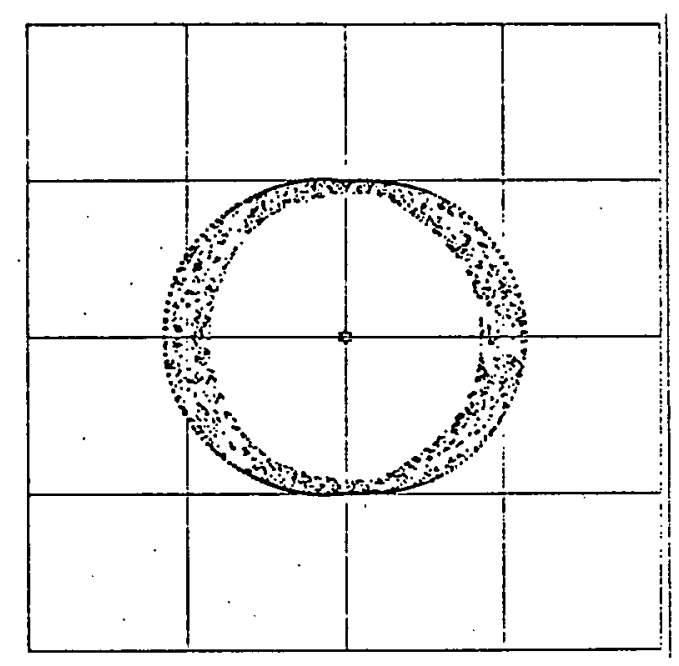

$$
\begin{aligned}
& B_{r 21}=.0014 \\
& B_{r 74}=.0006
\end{aligned}
$$

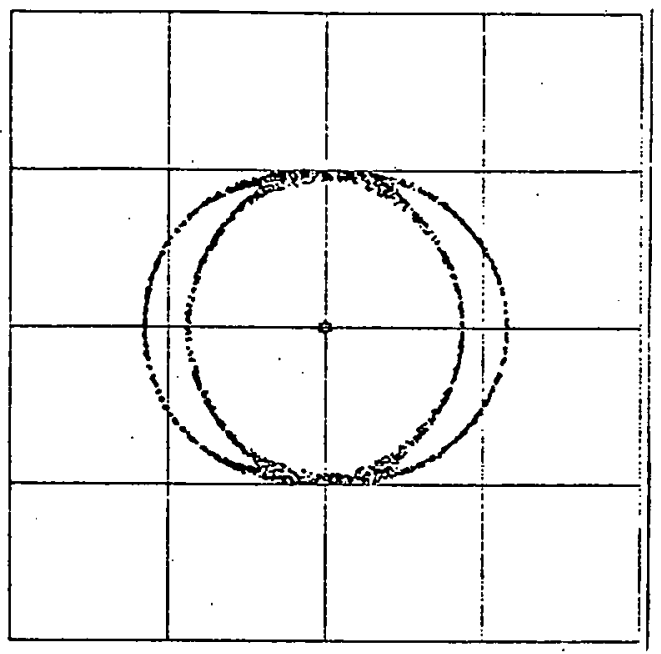

$$
\begin{aligned}
& \mathrm{B}_{\mathrm{r} 21}=.0018 \\
& \mathrm{~B}_{\mathrm{r} 74}=.0002
\end{aligned}
$$

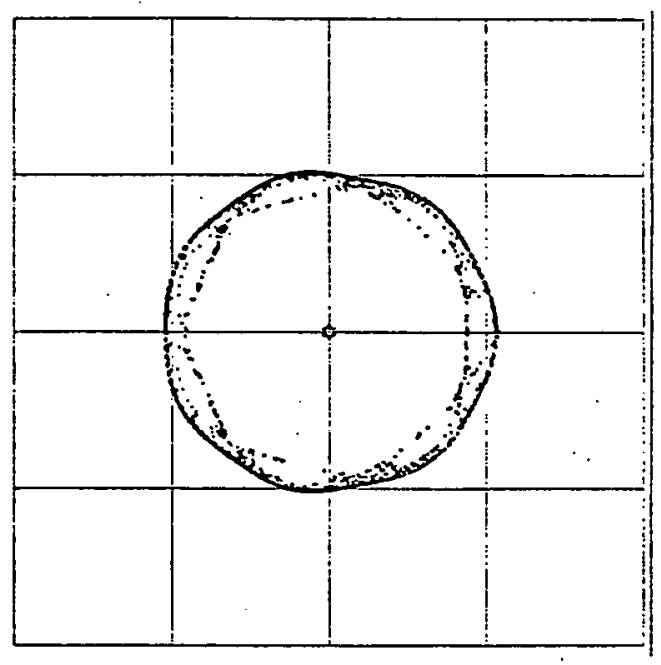

$$
\begin{aligned}
& \mathrm{B}_{\mathrm{r} 21}=.0002 \\
& \mathrm{~B}_{\mathrm{r} 74}=.0018
\end{aligned}
$$

FIG. 1. 

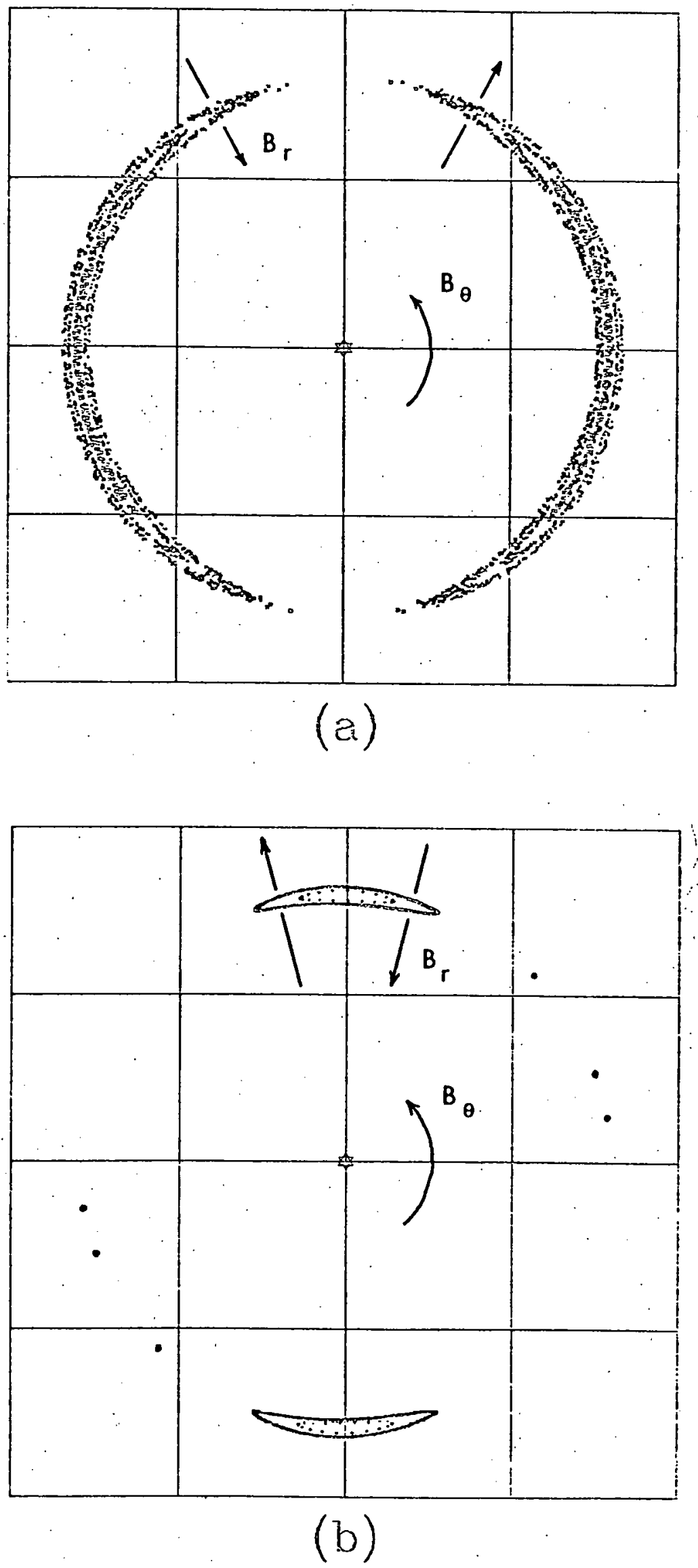

FIG. 2 


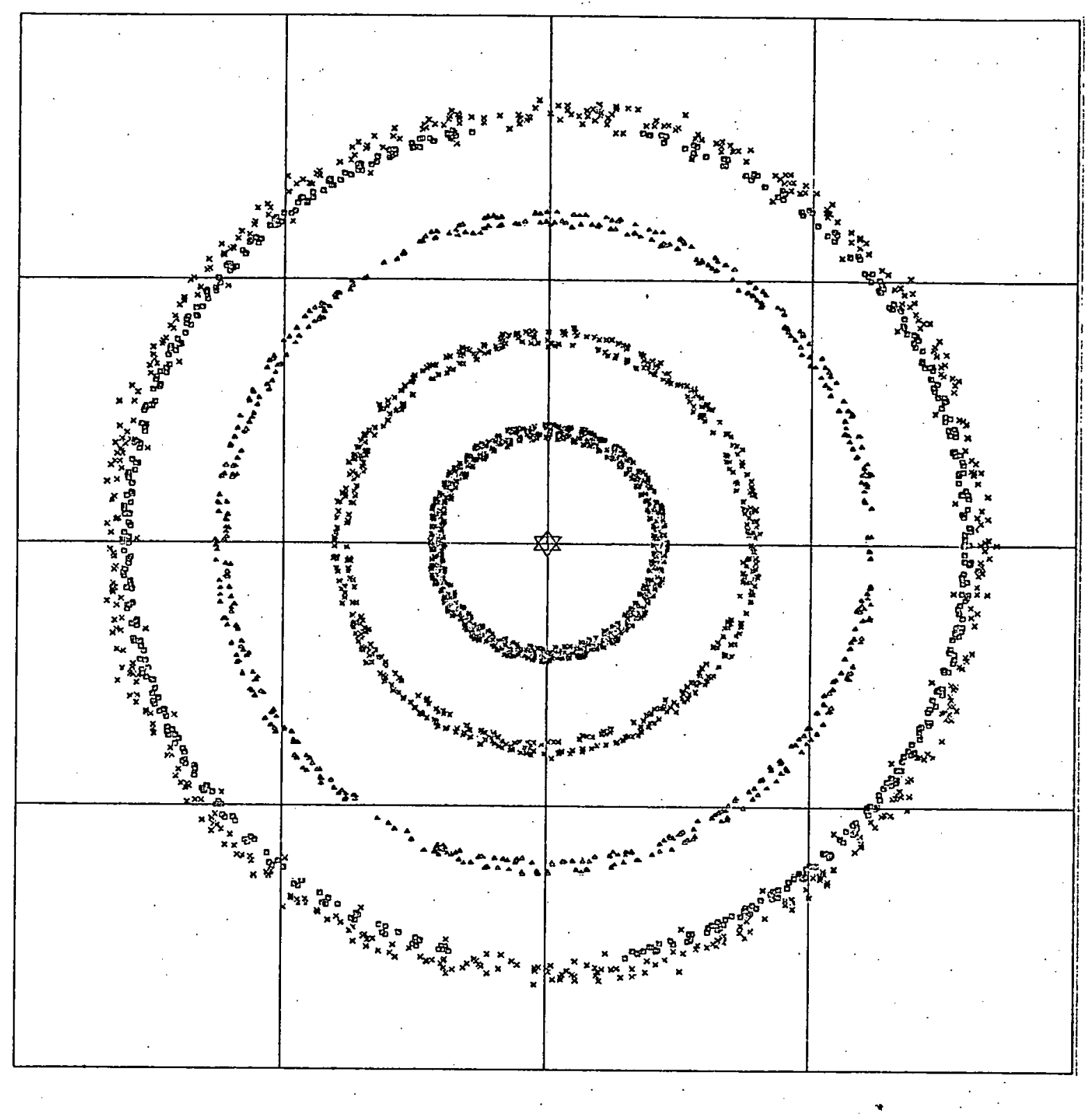

FIG. 3 . 
$\Delta^{0}$

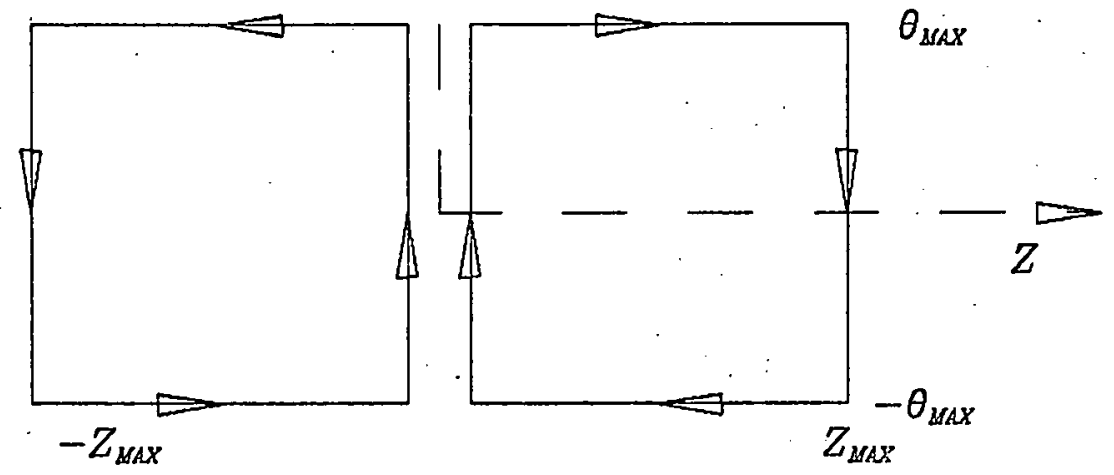

FIG. 4. 


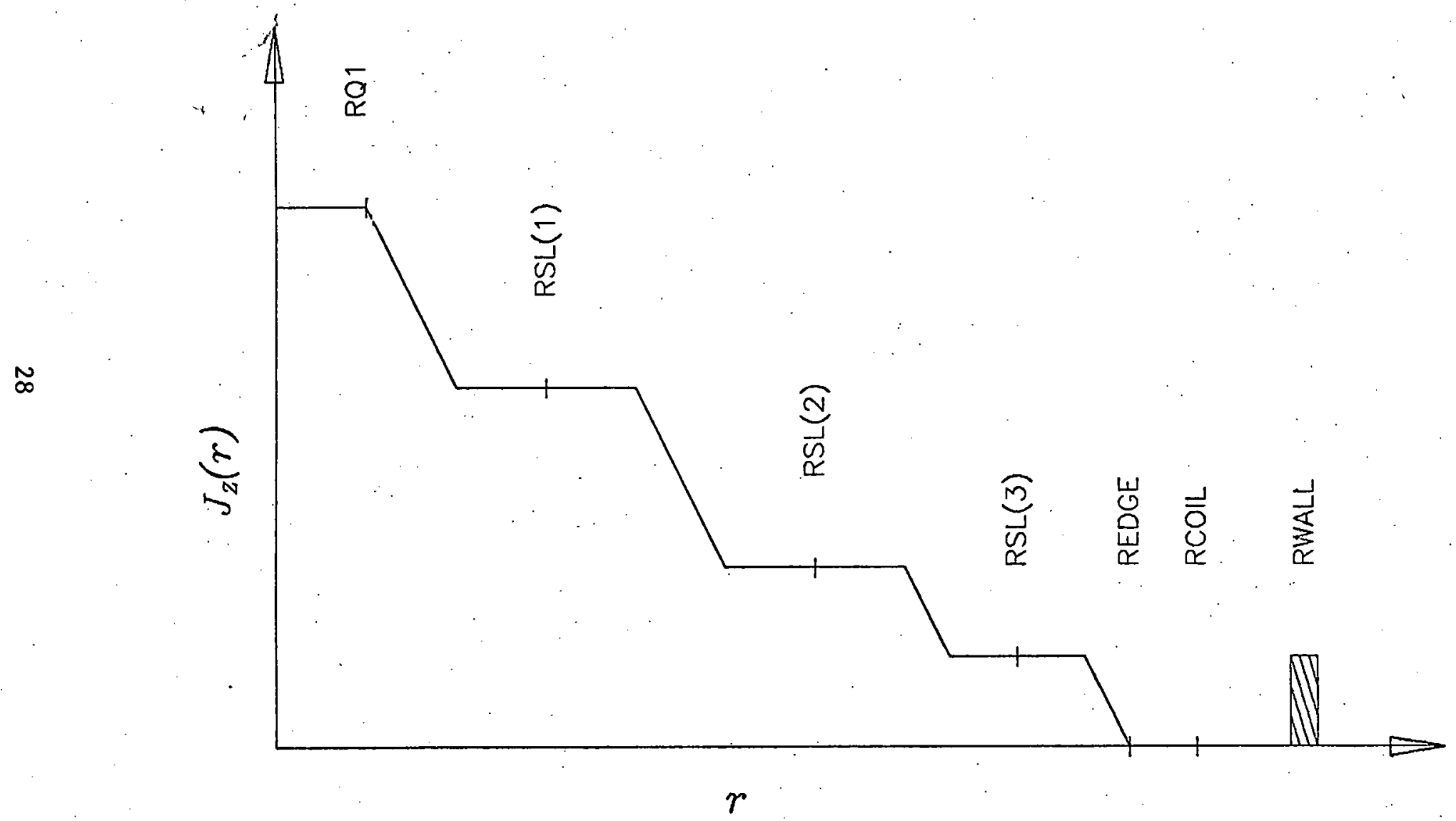

FIGURE $\mathbf{5}$ 


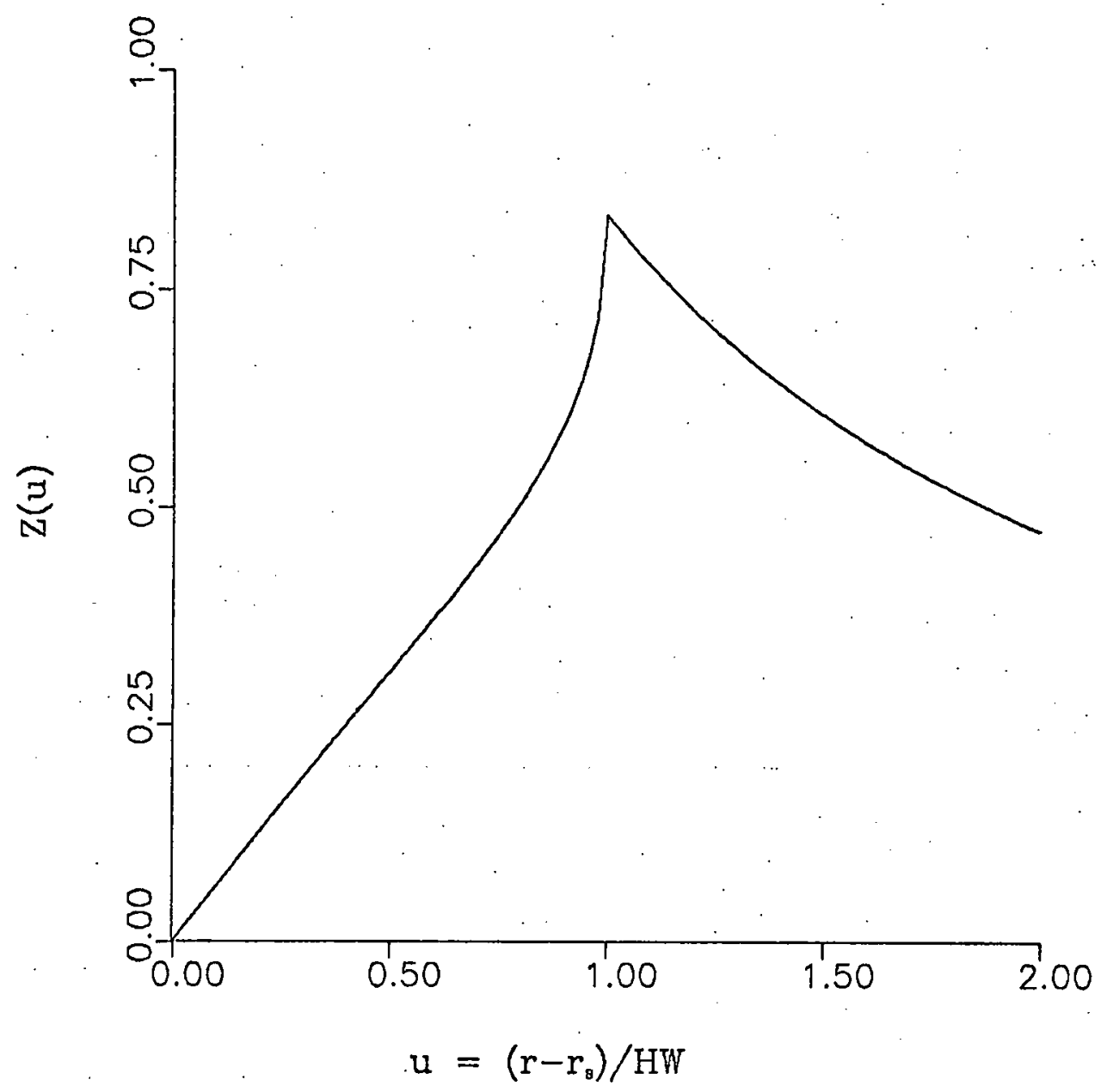

FIG. 6 


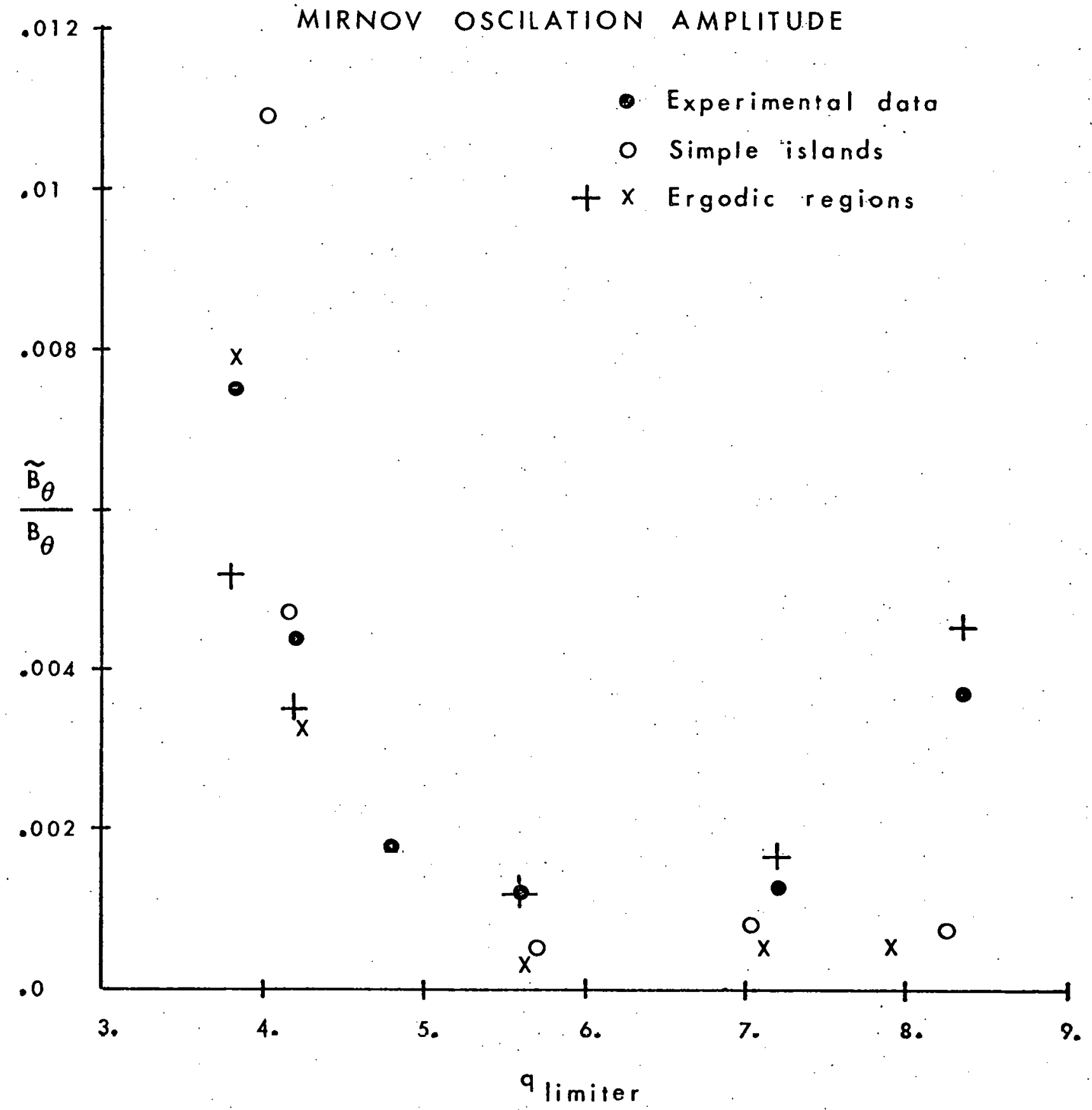

FIG 7 

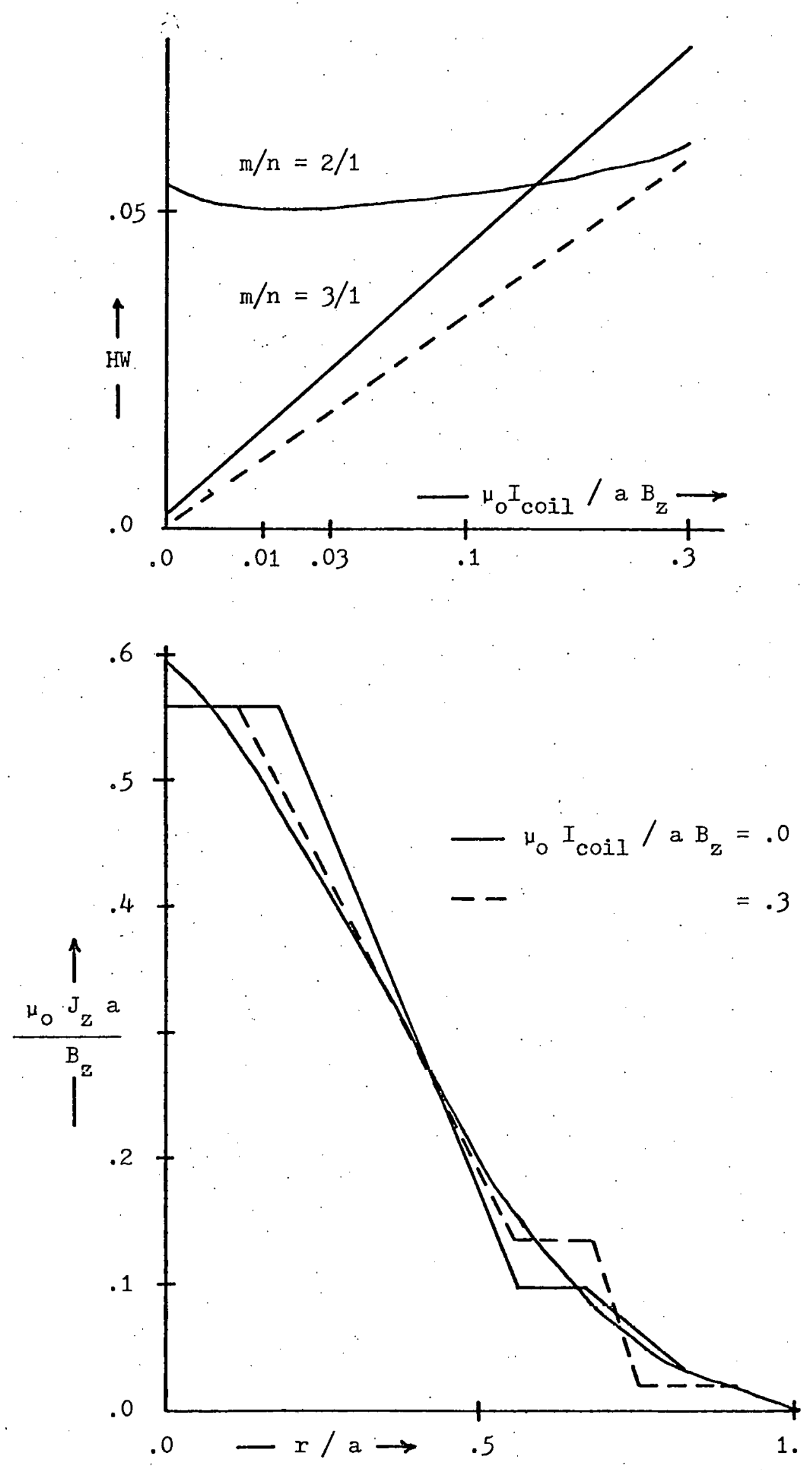

FIG. 8.

31 

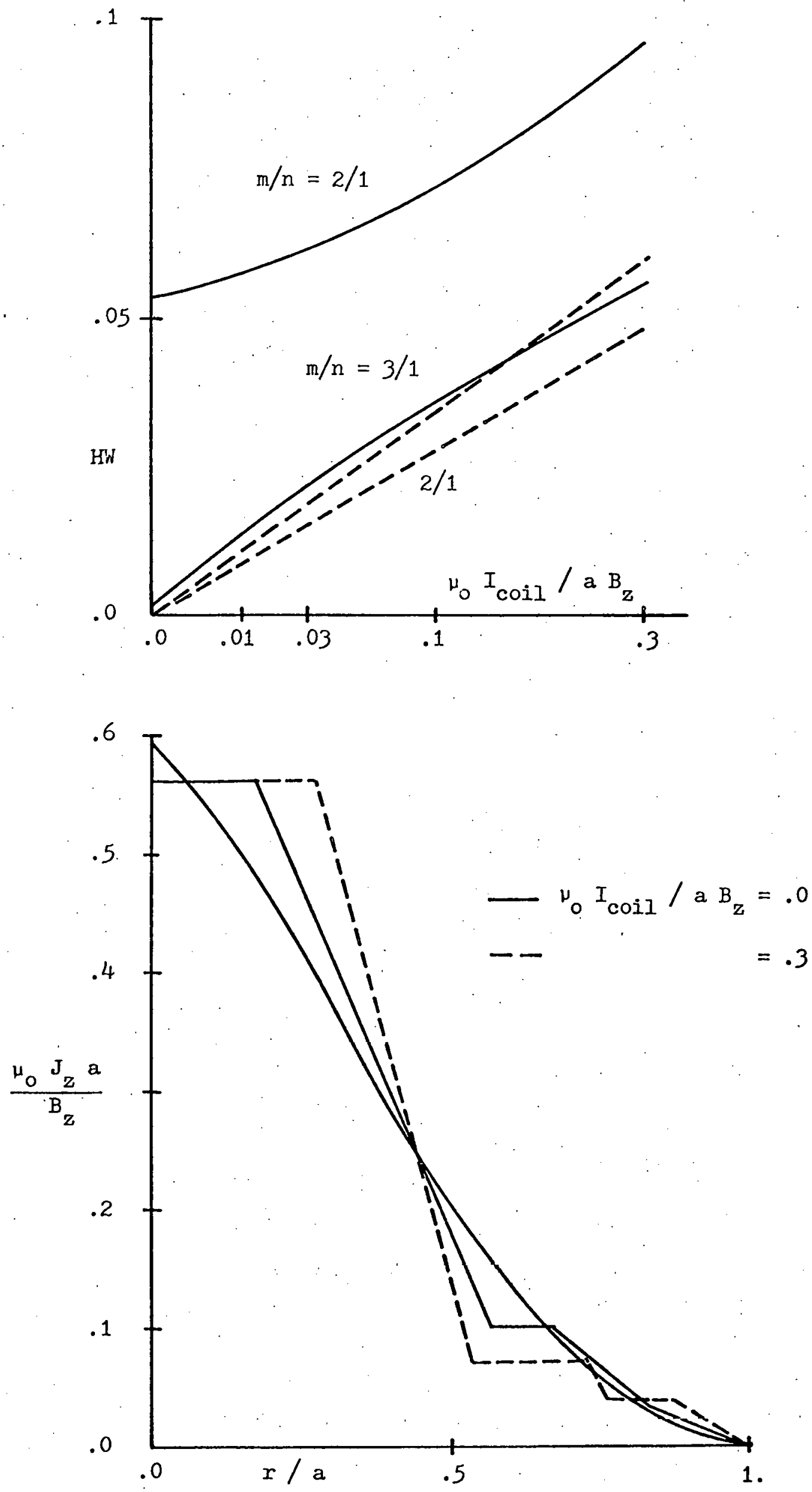


\section{REFERENCES}

[1] STOTT, P. E., C. M. Wilson and A. Gibson, Nucl. Fusion 17 (1977) 481, Part I, and Nucl. Fusion 18 (1978) 475, Part II.

[2] StotT, P. E., et al., in Plasma Wall Interaction (Proc. Int. Symp., Julich, 1976), Pergamon Press EUR 5782e, Oxford (1977) 39.

[3] SHEFF IELD J. and R. A. Dory, "The Ripple Bundle Divertor for Tokamaks," ORNL/TM-6220 (1978).

[4] Workshop on Physics and Engineering Problems of Divertors in Tokamak Reactors, (RUTHERFORD, P. H., ed.) Princeton Plasma Physics Laboratory (July 1979).

[5] WELLS, W. M., "ORNL TNS Program: Evaluation of the Bundle Divertor," ORNL/TM-67.27 (November 1979).

[6] STACEY, W. M., J. R. Gilleland, G. L. Kulcinski, P. H. Rutherford, et al., "INTOR: The U.S. Contribution to the International Tokamak Reactor Workshop 1979," IAEA (November 1979).

[7] LYON, J. F. and J. A. Rome, Bull. Am. Phys. Soc. 24 (1979) 1034.

[8] KARGER, F., et al., in Plasma Physics and Controlled Nuclear Fusion, IAEA (1975) Vol. 1,207 and IAEA (1977) Vol. $1,267$.

[9] LACKNER, K. and F. Karger, "Stabilization of Disruptive Instability by Resonant Helical Windings," Ann. Controlled Fusion. Theory Conf., San Diego (1977).

[10] Goldston, R. J. and H. H. Towner, Bull. Am. Phys. Soc. 24 (1979) 1045.

[11] RUTHERFORD, P. H., Phys. Fluids 16 (1973) 1903.

[12] WHITE, R. B., D. A. Monticello, M. N. Rosenbluth, B. V. Waddell, Phys. Hluids 20 (1977) 800 .

[13] CARRERAS, B, B. V. Waddell and H. R. Hicks, Nucl. Fusion 19 (1979) 1423. 
[14] BATEMAN, G., MHD Instabilities, The M.I.T. Press, Cambridge, 1978.

[15] ZWEBEN, S. J., Bull. Am. Phys. Soc. 24 (1979) 1072.

[16] CAllen, J. D., et al., in Plasma Physics and Controlled Nuclear Fusion Research 1978, IAEA (1979) Vol. $1,415$.

[17] VUILLEMIN M. and C. Gourdon, EUR-CEA-FC-393 (Fontenay-aux-Roses, France, 1967).

[18] CHRISMAN, P., J. Clarke and J. Rome, ORNL/TM-4501 (1974).

[19] FINN, J. M., Nucl. Fusion 15 (1975) 845.

[20] IMSL Library, Edition 7, International Math Science Library.

[21] BERRY M. V., in "Topics in Nonlinear Dynamics," (JORNA, S., ed.), (Am. Inst. Phys., New York, 1978) 16-120.

[22] FORSYTHE, G. E., M. A. Malcolm and C. B. Moler, Computer Methods for Mathematical Computations, Prentice-Hall, New Jersey, 1977.

[23] MURAKAMI, M. and J. L. Dunlap, private communication, 1980; ORNL/TM-5154(1976) 20.

[24] ENGELHARDT, W., et al., in Plasma Physics and Controlled Nuclear Fusion Research 1978, IAEA (1979) Vol. 1, 123. 